\title{
CORRIGENDUM
}

\section{Risk factors for sporadic Vibrio parahaemolyticus gastroenteritis in east China: a matched case-control study - CORRIGENDUM}

\author{
W. X. YAN, Y. DAI, Y.J. ZHOU, H. LIU, S.G. DUAN, H.H. HAN ANd Y. CHEN \\ doi: 10.1017/S0950268814001599, Published online by Cambridge University Press, 3 July 2014; \\ first published online 27 October 2014. \\ In the above mentioned article by Yan et al. [1], the authors would like to apply the following corrections regarding
} word-by-word citations. The corrections and locations are stated below.

\section{SUMMARY}

\section{The following sentences}

From the multivariable analysis, $V$. parahaemolyticus infections were associated with antibiotics taken during the 4 weeks prior to illness [odds ratio (OR) $8 \cdot 1,95 \%$ confidence interval (CI) $1 \cdot 2-56 \cdot 4$ )], frequent eating out (OR 3·3, 95\% CI 1·1-10·1), and shellfish consumption (OR $3 \cdot 2,95 \%$ CI $1 \cdot 0-9 \cdot 9$ ), with population-attributable fractions of $0 \cdot 09,0 \cdot 25$, and $0 \cdot 14$, respectively. Protective factors included keeping the aquatic products refrigerated (OR $0 \cdot 4,95 \%$ CI $0 \cdot 1-0 \cdot 9$ ) and pork consumption (OR 0.2, 95\% CI 0·1-0.8).

\section{should be replaced by}

From the multivariable analysis, $V$. parahaemolyticus infections were associated with antibiotics taken during the 4 weeks prior to illness [odds ratio (OR) 7·6, 95\% confidence interval (CI) 1·1-54·4)], frequent eating out (OR 3·3, 95\% CI 1·0-10·4), and shellfish consumption (OR 3·4, 95\% CI 1·0-11·1), with population-attributable fractions of $0 \cdot 09,0 \cdot 24$, and $0 \cdot 14$, respectively. Protective factors included keeping the aquatic products refrigerated (OR $0 \cdot 4,95 \% \mathrm{CI} 0 \cdot 2-1 \cdot 0)$ and pork consumption $(\mathrm{OR} 0 \cdot 2,95 \% \mathrm{CI} 0 \cdot 1-0 \cdot 9)$.

Page 3, in the left column, line 30.

Data collected included demographics; pre-existing illness; previous medication use; travel history (travel to other city/ province); animal contact; refrigeration of certain aquatic products (i.e. fish, shrimp, crab and shellfish, whether of freshwater or saltwater origin) at home; other family kitchen practices; food consumption; meals eaten outside of home; and drinking water source.

\section{should be replaced by}

Data collected included demographics; pre-existing illness; previous medication use; travel history (travel to other city/ province); animal contact; regular handling of raw meat; refrigeration of certain aquatic products (i.e. fish, shrimp, crab and shellfish, whether of freshwater or saltwater origin) at home; other family kitchen practices; food consumption; meals eaten outside of home; and drinking water source.

Page 3, in the right column, line 31.

In multivariable analysis, to determine the variables in the model we used forward elimination method.

\section{should be replaced by}

Multivariable analysis was carried out using forced entry method.

Page 5, in the left column, line 19.

In August, case-patients were four times more likely to eat shellfish compared to controls (OR 4.0, 95\% CI 1·2-13·3). However, no association was observed between illness and shellfish consumption in other months (OR 2.0, 95\% CI 0·4-9·9). 


\section{should be replaced by}

In August, case-patients were five times more likely to eat shellfish compared to controls (OR 5·3, 95\% CI 1·4-20 1). However, no association was observed between illness and shellfish consumption in other months (OR 1·5, 95\% CI 0·3-6.7).

\section{Page 5, in the left column, line 27.}

Factors associated with decreased risk were keeping the aquatic products refrigerated; and consumption of poultry other than chicken, pasteurized milk, pork, eggs, and nuts.

\section{should be replaced by}

Factors associated with decreased risk were keeping the aquatic products refrigerated; regular handling of raw meat; and consumption of poultry other than chicken, pasteurized milk, pork, eggs, and nuts.

\section{Page 5, in the right column, line 9.}

We found that cleaning kitchen counters with dishwashing liquid or detergent was more frequently reported by controls than by cases, although it did not reach statistical significance $(64 \cdot 8 \%$ vs. $56 \cdot 3 \%$; OR $0 \cdot 6,95 \%$ CI $0 \cdot 3-1 \cdot 1)$.

\section{should be replaced by}

We found that cleaning kitchen counters with dishwashing liquid or detergent was more frequently reported by controls than by cases, although it did not reach statistical significance $(64 \cdot 8 \%$ vs. $56 \cdot 3 \%$; OR $0 \cdot 6,95 \%$ CI $0 \cdot 3-1 \cdot 2)$.

\section{Page 5, in the right column, line 24 .}

In the final multivariable model, antibiotics taken in the 4 weeks prior to illness (OR $8 \cdot 1,95 \%$ CI $1 \cdot 2-56 \cdot 4$ ), eating out $\geq 3$ times (OR 3·3, 95\% CI 1·1-10·1), and shellfish consumption (OR 3·2, 95\% CI 1·0-9·9) were independent factors associated with an increased risk for illness (Table 4). Keeping the aquatic products refrigerated (OR $0 \cdot 4,95 \%$ CI $0 \cdot 1-0 \cdot 9$ ) and pork consumption (OR $0 \cdot 2,95 \%$ CI $0 \cdot 1-0 \cdot 8$ ) were independent factors associated with a reduced risk for infection. There was no association between eating poultry other than chicken in the 5 days before illness or interview and $V$. parahaemolyticus infection (OR $0 \cdot 5,95 \%$ CI $0 \cdot 2-1 \cdot 0)$ after adjusting for other risk factors.

\section{Should be replaced by}

In the final multivariable model, antibiotics taken in the 4 weeks prior to illness (OR $7 \cdot 6,95 \%$ CI $1 \cdot 1-54 \cdot 4$ ), eating out $\geq 3$ times (OR 3·3, 95\% CI 1·0-10·4), and shellfish consumption (OR 3·4, 95\% CI 1·0-11·1) were independent factors associated with an increased risk for illness (Table 4 ). Keeping the aquatic products refrigerated (OR $0 \cdot 4,95 \%$ CI $0 \cdot 2-1 \cdot 0)$ and pork consumption (OR $0 \cdot 2,95 \%$ CI $0 \cdot 1-0 \cdot 9$ ) were independent factors associated with a reduced risk for infection. There was no association between eating poultry other than chicken in the 5 days before illness or interview (OR $0 \cdot 5,95 \%$ CI $0 \cdot 2-1 \cdot 1)$ and regular handling of raw meat (OR $0 \cdot 2,95 \%$ CI $0 \cdot 0-1 \cdot 2)$ and $V$. parahaemolyticus infection after adjusting for other risk factors.

\section{Page 6, in the left column, line 16.}

The percentage of cases attributable to each of these risk factors was as follows: antibiotics taken in the 4 weeks prior to illness, 9\%; frequent eating out, 25\%; and shellfish consumption, 14\% (Table 3).

\section{should be replaced by}

The percentage of cases attributable to each of these risk factors was as follows: antibiotics taken in the 4 weeks prior to illness, $9 \%$; frequent eating out, 24\%; and shellfish consumption, $14 \%$ (Table 4 ). 
Table 1.

should be replaced by

Table 1. Socioeconomical characteristics of case-patients and controls enrolled in a population-based case-control study to identify risk factors for V. parahaemolyticus gastroenteritis, China, July 2010-June 2011

\begin{tabular}{|c|c|c|c|}
\hline Socioeconomical characteristics & $\begin{array}{l}\text { Cases }(n=71) \\
\text { No. }(\%)\end{array}$ & $\begin{array}{l}\text { Controls }(n=142) \\
\text { No. }(\%)\end{array}$ & $P$ value \\
\hline Education & & & $0 \cdot 430$ \\
\hline Primary school and lower & $7(9 \cdot 9)$ & $12(8 \cdot 5)$ & \\
\hline Secondary school & $16(22 \cdot 5)$ & $29(20 \cdot 4)$ & \\
\hline High school and above & $48(67 \cdot 6)$ & $101(71 \cdot 1)$ & \\
\hline Total family income per year & & & $0 \cdot 821$ \\
\hline $0-29999$ yuan* & $8(11 \cdot 3)$ & $20(14 \cdot 1)$ & \\
\hline $30000-79999$ yuan & $42(59 \cdot 2)$ & $80(56 \cdot 3)$ & \\
\hline$\geqslant 80000$ yuan & $17(23 \cdot 9)$ & $40(28 \cdot 2)$ & \\
\hline Refused to answer & $4(5 \cdot 6)$ & $2(1 \cdot 4)$ & \\
\hline
\end{tabular}

* 10 yuan $=0.95$ British pound sterling.

Table 3.

should be replaced by

Table 3. Univariable analysis of risk factors associated with V. parahaemolyticus gastroenteritis, China, July 2010-June 2011

\begin{tabular}{|c|c|c|c|}
\hline Risk factor & $\begin{array}{l}\text { Cases }(n=71) \\
\text { No. }(\%)\end{array}$ & $\begin{array}{l}\text { Controls }(n=142) \\
\text { No. }(\%)\end{array}$ & OR $(95 \% \mathrm{CI})$ \\
\hline Took antibiotics in the 4 weeks prior to illness & $7(9 \cdot 9)$ & $2(1 \cdot 4)$ & $7 \cdot 0(1 \cdot 5-33 \cdot 7)$ \\
\hline \multicolumn{4}{|l|}{ Frequency of eating out in the past 5 days } \\
\hline $1-2$ times & $25(35 \cdot 2)$ & $37(26 \cdot 1)$ & $2 \cdot 4(1 \cdot 2-4 \cdot 9)$ \\
\hline$\geqslant 3$ times & $25(35 \cdot 2)$ & $36(25 \cdot 4)$ & $3 \cdot 7(1 \cdot 4-9 \cdot 6)$ \\
\hline Ate shellfish in the past 5 days & $14(19 \cdot 7)$ & $13(9 \cdot 2)$ & $3 \cdot 1(1 \cdot 2-8 \cdot 1)$ \\
\hline Keep aquatic products refrigerated in the past 5 days & $25(35 \cdot 2)$ & $73(51 \cdot 4)$ & $0 \cdot 3(0 \cdot 2-0 \cdot 8)$ \\
\hline Regular handling of raw meat & $2(2 \cdot 8)$ & $13(9 \cdot 2)$ & $0 \cdot 3(0 \cdot 1-1 \cdot 2)$ \\
\hline Ate poultry other than chicken in the past 5 days & $20(28 \cdot 2)$ & $62(43 \cdot 7)$ & $0 \cdot 4(0 \cdot 2-0 \cdot 8)$ \\
\hline Consumed pasteurized milk in the past 5 days & $23(32 \cdot 4)$ & $69(48 \cdot 6)$ & $0 \cdot 4(0 \cdot 2-0 \cdot 8)$ \\
\hline Ate pork in the past 5 days & $63(88 \cdot 7)$ & $135(95 \cdot 1)$ & $0 \cdot 3(0 \cdot 1-1 \cdot 1)$ \\
\hline Ate eggs in the past 5 days & $47(66 \cdot 2)$ & $131(92 \cdot 3)$ & $0 \cdot 1(0 \cdot 0-0 \cdot 3)$ \\
\hline Ate nuts in the past 5 days & $10(14 \cdot 1)$ & $65(45 \cdot 8)$ & $0 \cdot 1(0 \cdot 0-0 \cdot 2)$ \\
\hline
\end{tabular}

OR, Odds ratio; CI, Confidence interval. 
Table 4.

should be replaced by

Table 4. Multivariable analysis of risk factors associated with V. parahaemolyticus gastroenteritis, China, July 2010-June 2011

\begin{tabular}{ll}
\hline \hline Risk factor & OR $(95 \%$ CI $)$ \\
\hline Took antibiotics in the 4 weeks prior to illness & $7 \cdot 6(1 \cdot 1-54 \cdot 4)$ \\
Frequency of eating out in the past 5 days & $1 \cdot 7(0 \cdot 7-4 \cdot 0)$ \\
$1-2$ times & $3 \cdot 3(1 \cdot 0-10 \cdot 4)$ \\
$\geqslant 3$ times & $3 \cdot 4(1 \cdot 0-11 \cdot 1)$ \\
Ate shellfish in the past 5 days & $0 \cdot 5(0 \cdot 2-1 \cdot 1)$ \\
Ate poultry other than chicken in the past 5 days & $0 \cdot 4(0 \cdot 2-1 \cdot 0)$ \\
Keep aquatic products refrigerated in the past 5 days & $0 \cdot 2(0 \cdot 1-0 \cdot 9)$ \\
Ate pork in the past 5 days & $0 \cdot 2(0 \cdot 0-1 \cdot 2)$ \\
Regular handling of raw meat & $13 \cdot 9$ \\
\hline \hline
\end{tabular}

OR, Odds ratio; CI, Confidence interval; PAF, Population-attributable fraction.

\section{REFERENCE}

1. Yan WX, et al. Risk factors for sporadic Vibrio parahaemolyticus gastroenteritis in east China: a matched case-control study. Epidemiology and Infection, Published by Cambridge University Press 3 July 2014, doi:10.1017/S0950268814001599 